J. clin. Path., 1977, 30, 467-472

\title{
Skeletal muscle pathology in chronic heart block
}

\author{
C. D. LAMBERT AND A. J. FAIRFAX \\ From the Department of Neurology, St. George's Hospital, London SWI and Department of Medicine, \\ Middlesex Hospital, London W1
}

SUMMARY Skeletal muscle samples, mainly from the deltoid, were studied morphologically and histochemically in 35 patients with chronic heart block and from nine elderly control subjects. The average age of the first group was 67.7 (range 11-94) years. Abnormalities were present in 20 cases, no difference being found between patients with idiopathic and secondary heart block. In 15 samples there was increased oxidative enzyme activity in some muscle fibres, and in six there was fibre type grouping. Six had unexplained type II fibre atrophy and two had a predominance of type I fibres. Tubular aggregates were conspicuous in one biopsy specimen, and in another, rod bodies were found. Minor abnormalities were also seen in the control group. In the absence of any consistent pattern many of these changes were attributed to ageing.

Cardiac conduction disturbances are a recognised feature of some neurological disorders, and complete heart block has been described in association with dystrophia myotonica (Litchfield, 1953), with scapuloperoneal dystrophy (Mawatari and Katayama, 1973), and with the oculocranio somatic syndrome (Kearns and Sayre, 1958; Olson et al., 1972). A recent survey of a large group of patients with chronic heart block attending cardiac clinics showed that such cases were rare, and, in the remainder, neurological assessment shed no light on the aetiology of the conduction disturbance (Lambert and Fairfax, 1976).

Postmortem studies of cases of chronic heart block have demonstrated that the most common finding is fibrosis selectively replacing the Purkinje fibres, and this has been described as a 'myopathy' of the conducting system (Lenègre, 1964). In animals, some histochemical similarities exist between the Purkinje fibres of the heart and the type II fibres of skeletal muscle (Snijder and Meijer, 1970). Skeletal muscle has not, however, been systematically studied in patients with chronic heart block. Muscle morphology and histochemistry were therefore examined in a series of patients with complete heart block to determine whether any abnormality of skeletal muscle was present in these cases.

\section{Patients and methods}

Thirty-five consecutive patients admitted for pace-

Received for publication 6 October 1976 maker implantation with chronic atrioventricular block were studied. Informed consent was obtained from each subject. A full neurological assessment was made and serum creatine phosphokinase was measured. A skeletal muscle specimen was taken under local anaesthesia, usually from the deltoid muscle, during implantation of the pacemaker. The sample was processed using conventional techniques (Dubowitz and Brooke, 1973). Morphological detail was demonstrated by the modified Gomori trichrome method (Engel and Cunningham, 1963); the histochemical methods used were: adenosine triphosphatase reaction at $\mathrm{pH} 9.4$ (Padykula and Herman, 1955); succinic dehydrogenase (Nachlas et al., 1957); NADH-tetrazolium reductase (Novikoff et al., 1961); phosphorylase (Takeuchi and Kuriaki, 1955); periodic acid Schiff (PAS), and Sudan black.

A control series of deltoid muscle specimens was obtained as soon as possible post mortem from nine patients aged over 65 years who had died from causes other than heart block.

\section{Results}

The 35 patients studied, 24 men and 11 women, were aged between 11 and 94 with a mean of $67 \cdot 7$ years. In 11 cases a recognised cause of heart block was present. These included: aortic valve disease (3), cardiomyopathy (2), familial heart block (2), and congenital heart block (1). There were also single cases of chronic heart block secondary to ischaemic heart disease, to sarcoidosis, and to septal 
calcification in association with Paget's disease. In the remaining 24 patients the disorder was idiopathic.

No examples of those neurological disorders known to be associated with heart block were identified in this series, but unrelated neurological abnormalities were found in 21 patients including both those with idiopathic and those with secondary heart block. Twenty of these patients were aged 60 years or more. The commonest abnormalities were: cervical spondylosis (5), deafness (4), absent ankle reflexes (4), sensory impairment of the legs (3), impaired upward gaze (3), and ptosis (2). There were single examples of Parkinson's disease, trigeminal neuralgia, and traumatic hemiparesis.

Serum creatine phosphokinase levels were normal in 28 out of 29 patients, and in the only subject with a raised level (284 IU/1) no neurological abnormality was found.

\section{SKELETAL MUSCLE MORPHOLOGY}

Morphological examination of the 35 muscle specimens from patients with chronic heart block showed no microscopic evidence of muscular dystrophy. There was also no inflammatory infiltration present in any of the specimens. The patient with sarcoidosis had a normal muscle biopsy although sarcoid granulomata were present in skin lesions and there was evidence of pulmonary, myocardial, and visceral involvement.

The proportion of type II fibres in the deltoid muscle specimens varied from 17 to $66 \%$ with a

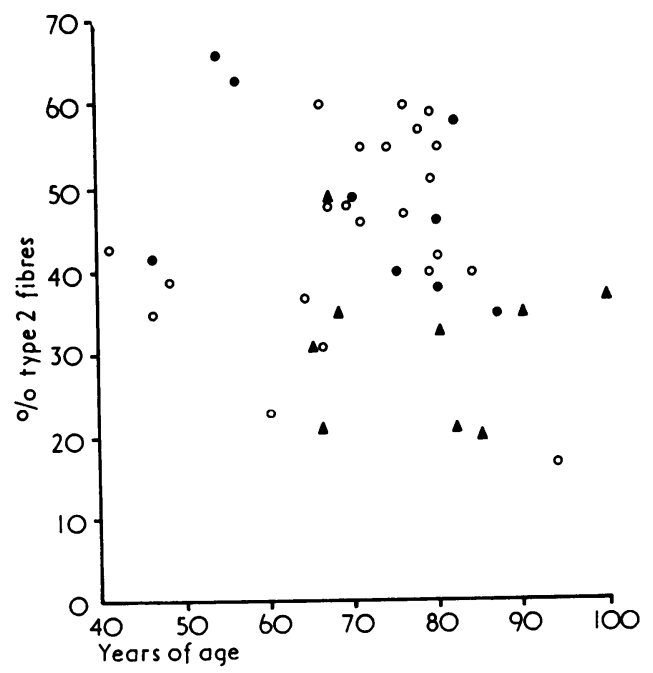

Fig. 1 Proportion of type II fibres of deltoid muscle in idiopat hic (open circles) and secondary (closed circles) heart block and in elderly controls (triangles). mean of $46.0 \% \pm 11.5$ (SD). The mean percentage of type II fibres in the postmortem control series was $31 \cdot 3 \% \pm 9 \cdot 5$ (SD) with a range of $20-49 \%$ (Fig. 1). In two patients with idiopathic heart block and in three controls, the proportion of type II fibres was less than $24 \%$, quoted as the lower $95 \%$ confidence limit for this muscle in young adults (Johnson et al., 1973).

Fifteen of the 35 muscle specimens were normal, 10 of these being from patients under 70 years of age. In the remaining 20 biopsy specimens one or more abnormalities were found (Table).

In 15 patients (14 of whom were over 70 years of age) increased subsarcolemmal staining was demonstrable in some fibres using the succinic dehydrogenase reaction (Fig. 2) and some of these appeared similar to 'ragged-red' fibres (Engel, 1971) using the Gomori trichrome stain. Increased staining of these abnormal fibres was seen with Sudan black (Fig. 3). In none of the specimens did 'ragged-red' fibres comprise more than $1 \%$ of the total muscle fibres.

Rod bodies were seen in some fibres (Fig. 4) in an 80-year-old man with calcific aortic valve disease, Paget's disease, and polymyalgia rheumatica treated by steroids; increased subsarcolemmal staining and type II fibre atrophy were also present.

Tubular aggregates (Fig. 5) were identified in $20 \%$ of the skeletal muscle fibres in a 62 -year-old man with unexplained cardiomyopathy and aortic incompetence. He had complained of recurrent leg cramps and on examination showed slight wasting and weakness of both quadriceps femoris muscles. Tubular aggregates were present in this muscle and were also found three months later in the pectoralis major. Further samples were obtained on a third occasion during an operation for aortic valve replacement: the rectus abdominis and muscle from the atria and ventricles were examined histochemically $ᄋ$ and by electron microscopy (Dr D. Landon) but $>$ tubular aggregates could not be identified in any of these specimens.

Six muscle biopsy specimens ( 3 from patients with $\Omega$ cervical spondylosis) showed grouping of fibre $N$

Table Skeletal muscle abnormalities found in 20 specimens from patients with chronic complete heart block

Irregular distribution of oxidative enzyme activity

Type grouping

Type 2 fibre atrophy

Type 1 fibre predominance

Rod bodies

Tubular aggregates

Central nuclei increased 


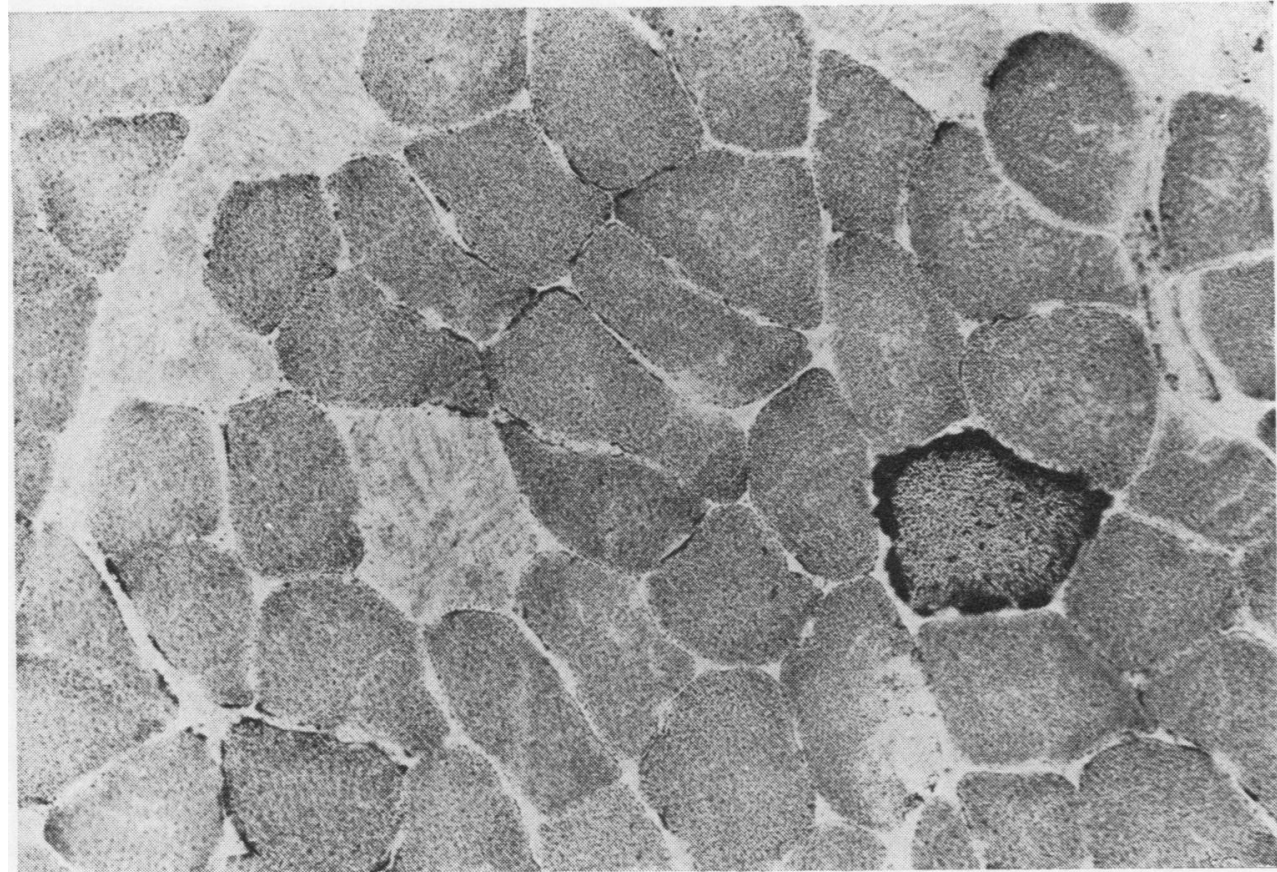

Fig. 2 Deltoid muscle from a patient with idiopathic heart block showing increased subsarcolemmal staining by the succinic dehydrogenase reaction $(\times 180)$.

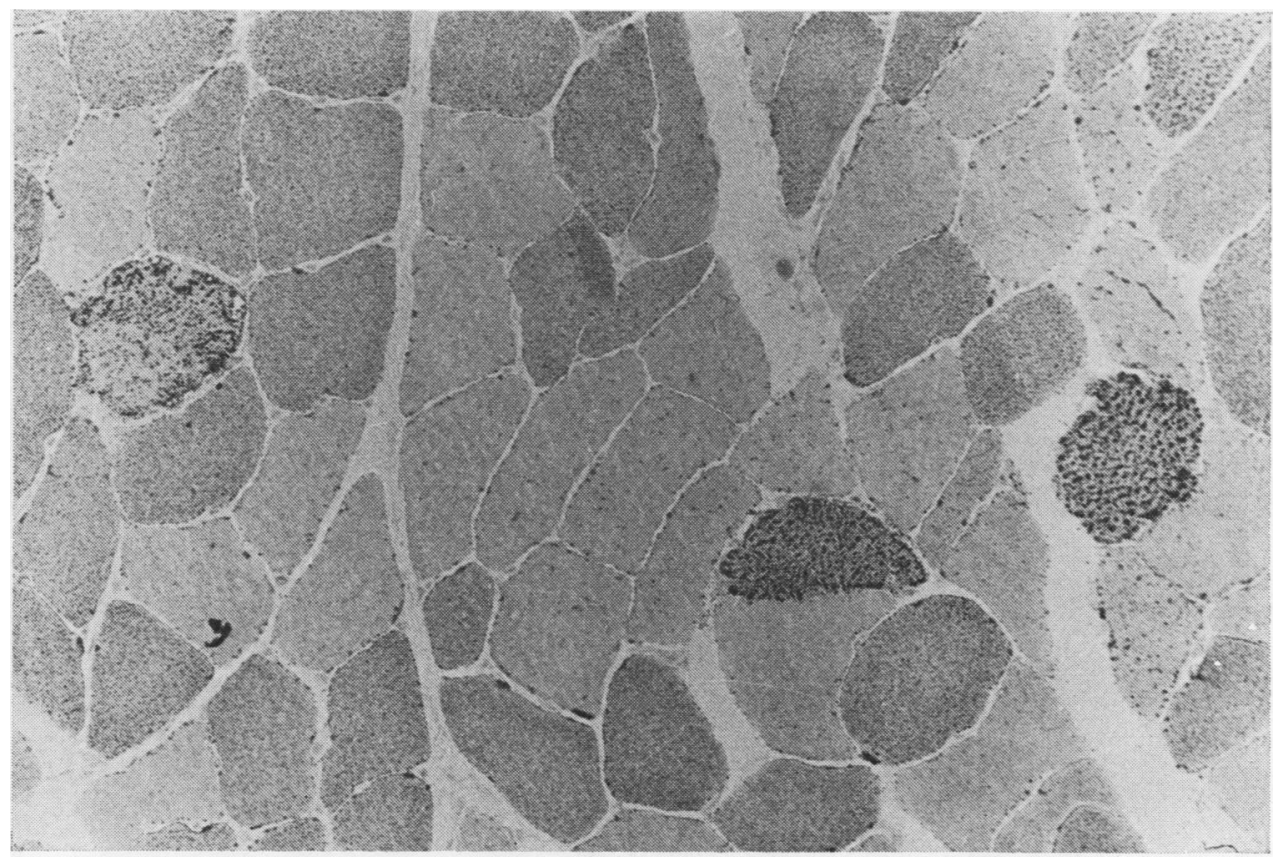

Fig. 3 Abnormal fibres in the deltoid muscle of the same patient using the Sudan black stain $(\times 110)$. 


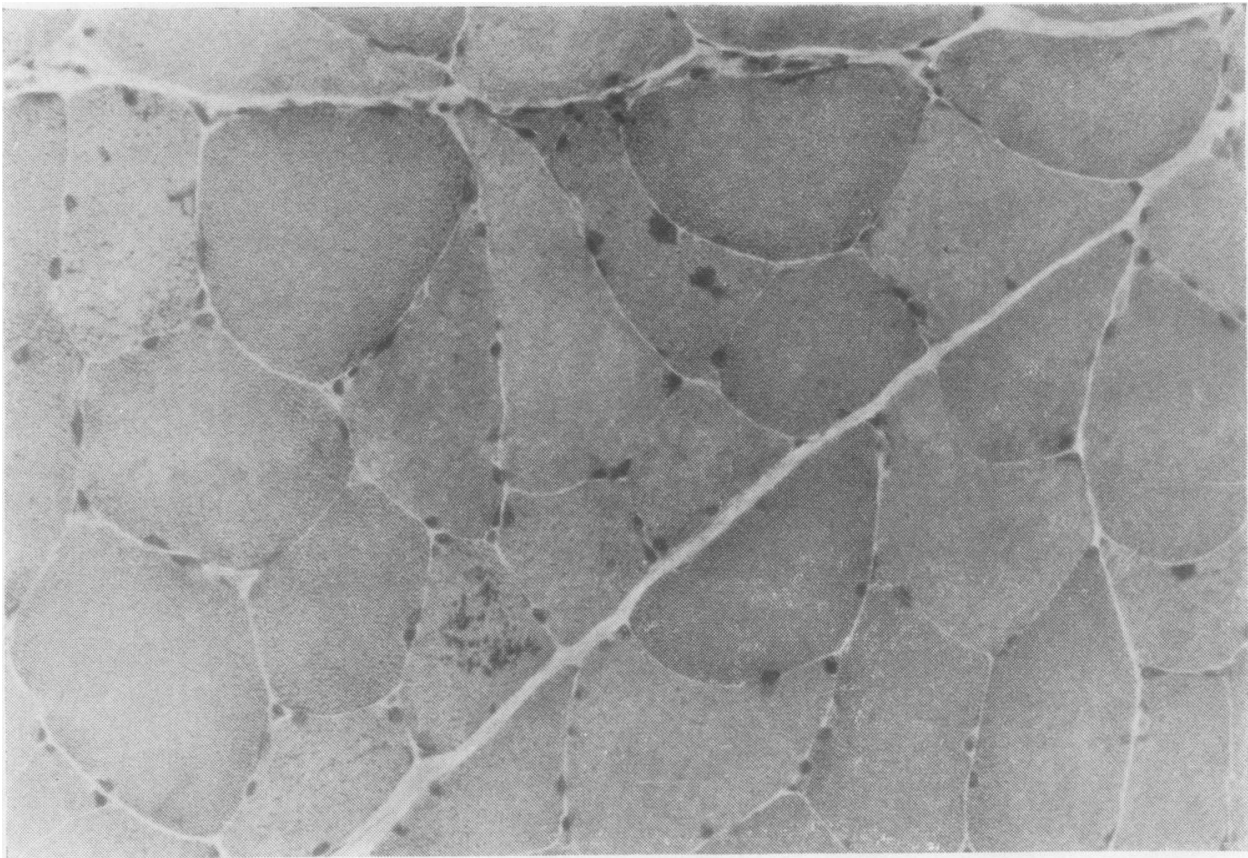

Fig. 4 Rod bodies present in deltoid muscle of a patient with secondary heart block and polymyalgia rheumatica $($ Trichrome stain $\times 280$ ).

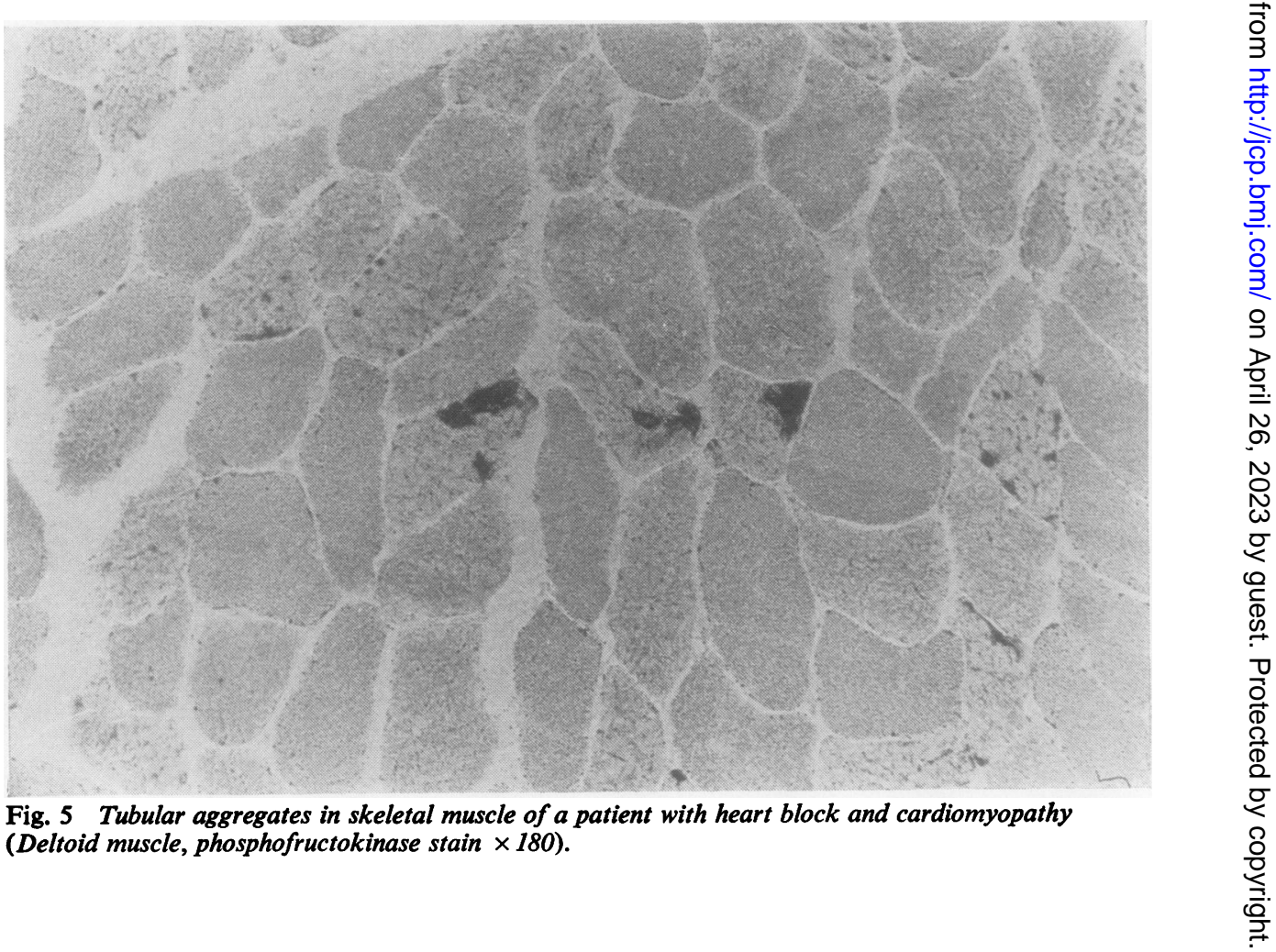


types. Six other specimens, including two from patients with aortic valve disease, showed unexplained atrophy of type II fibres. Increased central nuclei $(15 \%)$ were the sole abnormality found in deltoid muscle from a 46-year-old man with vitiligo and chronic heart block possibly of autoimmune aetiology (Fairfax and Leatham, 1975).

In the postmortem series satisfactory fibre typing could be obtained up to 48 hours after death using the ATPase reaction, but poor results were obtained using the other histochemical methods, and morphological detail was not satisfactorily demonstrated. The specimens examined all showed various abnormalities, including type I fibre predominance, type II fibre atrophy, fibre-type grouping, and irregular subsarcolemmal staining for oxidative enzymes.

\section{Discussion}

Patients with heart block in association with overt neuromuscular disease form a clinically recognisable small group which accounts for less than $1 \%$ of all cases of chronic atrioventricular block (Lambert and Fairfax, 1976). This previous study, and a review of the literature, indicates that these neurological diseases are predominantly nultisystem disorders, such as dystrophia myotonica, rather than the 'true' muscular dystrophies. For example, in the oculocraniosomatic syndrome, in addition to ophthalmoplegia and muscular weakness there is ataxia, pigmentary retinal degeneration, nerve deafness, and shortness of stature (Drachman, 1968). Mitochondrial abnormalities have been described affecting sweat glands (Karpati et al., 1973), cerebellum (Schneck et al., 1973), and skeletal muscle (Olson et al., 1972), the latter giving rise to the 'ragged-red' appearance with the trichrome stain.

In the present study the finding of morphological abnormalities of skeletal muscle in secondary heart block and in the control samples led us to the conclusion that the changes observed in patients with idiopathic heart block are unrelated to the aetiology of their conduction disturbance. Furthermore, although there are some histochemical similarities between type II skeletal muscle fibres and the Purkinje tissue (Snijder and Meijer, 1970), no specific abnormality of type II muscle fibres has been identified in this study. Immunochemically, the Purkinje tissue has recently been shown to be more comparable to cardiac muscle and to the type I fibres of skeletal muscle than to type II fibres (Fairfax and Doniach, 1976).

This study is the most comprehensive survey of the morphology and histochemistry of skeletal muscle from elderly living subjects. It indicates that abnormalities of skeletal muscle are common in this age group.

Our pathological results are similar to those noted in a postmortem study of skeletal muscle in the elderly (Jennekens et al., 1971): these authors attributed the changes observed to disuse, inadequate nutrition, myopathic change of undetermined cause, and neurogenic factors. Our subjects were active and well nourished. Careful examination gave no indication of any clinical myopathy but minor neurological abnormalities and multiple disease processes were common. This makes it difficult to evaluate the changes found in the skeletal muscle of elderly people, even in the context of detailed clinical and biochemical information.

In those patients in whom discrete abnormalities were identified, their significance remains uncertain. Tubular aggregates, which are known to occur in hypertrophic myocardium (Maron and Ferrans, 1974), have been described in skeletal muscle in a number of clinical conditions, including one elderly subject with cramps and muscle stiffness (MorganHughes et al., 1970). Similarly, rod bodies were originally described in association with a mild non-progressive myopathy of infancy (Shy et al., 1963), but these have subsequently been identified in other disorders, including the collagen diseases (Engel, 1967). It is interesting, therefore, that our patient had polymyalgia rheumatica.

Ultrastructural studies have shown that increased staining for succinic dehydrogenase, one of the oxidative mitochondrial enzymes, usually corresponds to the presence of increased numbers of mitochondria, and these may also show morphological and biochemical abnormalities (Afifi et al., 1972). Abnormal mitochondria and lipid accumulations patchily distributed in skeletal muscle are a consistent feature of oculocraniosomatic syndrome in which heart block occurs (Olson et al., 1972). The frequent finding of these changes in muscle from elderly, but not from younger subjects suggests that this phenomenon may also be a feature of ageing.

We thank Dr H. Johnson, St. Thomas' Hospital, for providing postmortem material; Dr D. Landon, National Hospital for Nervous Diseases, for electron microscopy; and Dr A. Leatham for encouragement in the study. We also thank Mrs J. Batts, Miss M. Ellison, and Miss R. J. Biles for technical and secretarial assistance.

\section{References}

Afif, A. K., Ibrahim, M. Z. M., Bergman, R. A., Haydar, 
N. A., Mire, J., Bahuth, N., and Kaylani, F. (1972). Morphologic features of hypermetabolic mitochondrial disease. A light microscopic, histochemical and electron microscopic study. J. Neurol. Sci., 15, 271290.

Drachman, D. A. (1968). Ophthalmoplegia plus. The neurodegenerative disorders associated with progressive external ophthalmoplegia. Arch. Neurol. (Chic.), 18, 654-674.

Dubowitz, V. and Brooke, M. H. (1973). Muscle Biopsy: A Modern Approach. W. B. Saunders, London.

Engel, W. K. (1967). A critique of congenital myopathies and other disorders. In Exploratory Concepts in Muscular Dystrophy and Related Disorders, edited by A. T. Milhorat. I.C.S., 147, pp. 27-40. Excerpta Medica Foundation, Amsterdam.

Engel, W. K. (1971). 'Ragged-red' fibres in ophthalmoplegia syndromes and their differential diagnosis. In 2nd International Congress on Muscle Diseases, edited by B. A. Kakulas. Abstract 72. Excerpta Medica, Amsterdam.

Engel, W. K. and Cunningham, G. C. (1963). Rapid examination of muscle tissue. An improved trichrome method for fresh-frozen biopsy sections. Neurology (Minneap.), 13, 919-923.

Fairfax, A. J. and Doniach, D. (1976). Autoantibodies to cardiac conducting tissue and their characterisation by immunofluorescence. Clin. exp. Immunol., 23, 1-8.

Fairfax, A. J. and Leatham, A. (1975). Idiopathic heart block: association with vitiligo, thyroid disease, pernicious anaemia, and diabetes mellitus. Brit. med. J., 4, 322-324.

Jennekens, F. G. I., Tomlinson, B. E., and Walton, J. N. (1971). Histochemical aspects of five limb muscles in old age. An autopsy study. J. neurol. Sci., 14, 259-276.

Johnson, M. A., Polgar, J., Weightman, D., and Appleton, D. (1973). Data on the distribution of fibre types in thirty-six human muscles. An autopsy study. J. Neurol. Sci., 18, 111-129.

Karpati, G., Carpenter, S., Larbrisseau, A., and Lafontaine, R. (1973). The Kearns-Shy syndrome. A multisystem disease with mitochondrial abnormality demonstrated in skeletal muscle and skin. J. Neurol. Sci., 19, 133-151.

Kearns, T. P. and Sayre, G. P.(1958). Retinitis pigmentosa, external ophthalmoplegia, and complete heart block. Unusual syndrome with histologic study in one of two cases. Arch. Ophthal., 60, 280-289.

Lambert, C. D. and Fairfax, A. J. (1976). Neurological associations of chronic heart block. J. Neurol. Neuro- $\overrightarrow{\vec{F}}$ surg. Psychiat., 39, 571-575.

Lenègre, J. (1964). Aetiology and pathology of bilateral bundle branch block in relation to complete heart block. Progr. Cardiovasc. Dis., 6, 409-444.

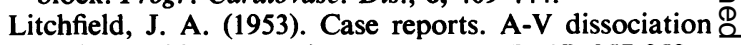
in dystrophia myotonica. Brit. Heart J., 15, 357-359.

Maron, B. J. and Ferrans, V. J. (1974). Aggregates of ${ }^{\text {क }}$ tubules in human cardiac muscle cells. J. Molec. cell. $\vec{\circ}$ Cardiol., 6, 249-264.

Mawatari, S. and Katayama, K. (1973). Scapuloperoneal muscular atrophy with cardiopathy. Arch. Neurol. $\frac{}{8}$ (Chic.), 28, 55-59.

Morgan-Hughes, J. A., Mair, W. G. P., and Lascelles, P. T. (1970). A disorder of skeletal muscle associated or with tubular aggregates. Brain, 93, 873-880.

Nachlas, M. M., Tsou, K. C., DeSouza, E., Cheng, C. S., $\stackrel{\odot}{\checkmark}$ and Seligman, A. M. (1957). Cytochemical demonstra-응 tion of succinic dehydrogenase by the use of a new p-nitrophenyl substituted ditetrazole. J. Histochem. Cytochem., 5, 420-436.

Novikoff, A. B., Shin, W. Y., and Drucker, J. (1961). Mitochondrial localization of oxidative enzymes: $\overrightarrow{0}$ staining results with two tetrazolium salts. J. biophys. $\checkmark$ biochem. Cytol., 9, 47-61.

Olson, W., Engel, W. K., Walsh, G. O., and Einaugler, R. (1972). Oculocraniosomatic neuromuscular disease with 'ragged-red' fibers. Arch. Neurol. (Chic.), 26, $\overline{\mathrm{O}}$ 193-211.

Padykula, H. A. and Herman, E. (1955). The specificity of the histochemical method for adenosine triphos- $\vec{F}$ phatase. J. Histochem. Cytochem., 3, 170-195.

Schneck, L., Adachi, M., Briet, P., Wolintz, A., and Volk, B. W.(1973). Ophthalmoplegia plus with morphological and chemical studies of cerebellar and muscle tissue. J. neurol. Sci., 19, 37-44.

Shy, G. M., Engel, W. K., Somers, J. E., and Wanko, T. (1963). Nemaline myopathy: a new congenital myopathy. Brain, 86, 793-810.

Snijder, J. and Meijer, A. E. F. H. (1970). Enzyme histochemical studies on Purkinje fibres of canine, bovine and porcine hearts. Histochem. J., 2, 395-409.

Takeuchi, T. and Kuriaki, H. (1955). Histochemicab detection of phosphorylase in animal tissues. J. Histo chem. Cytochem., 3, 153-160. 